Ethical and Regulatory Issues with Conducting Sexuality Research with LGBT Adolescents: A Call to Action for a Scientifically Informed Approach

\title{
Brian Mustanski
}

Address correspondence to:

Brian Mustanski, Ph.D.

IMPACT LGBT Health and Development Program

University of Illinois at Chicago

1747 W. Roosevelt Rd (M/C 747)

Chicago, IL 60608

brian@impactprogram.org 


\begin{abstract}
Lesbian, gay, bisexual, and transgender (LGBT) adolescents experience disparities in mental and sexual health. There is also a lack of research on this population relative to other adolescents, which limits our ability to effectively address these health disparities. Researchers may unfortunately avoid conducting research with this population because of anticipated or actual experiences with difficulties in obtaining IRB approval. A case example is provided to illustrate the ethical and regulatory issues related to research with LGBT adolescents. Relevant U.S. federal and local regulations related to research on sexual and mental health with adolescents is then reviewed. Data are presented demonstrating that requiring parental consent for LGBT youth under age 18 would likely alter study result. Data are also presented on participants' appraisals of the risks and discomforts associated with research participation. The provision of such empirical data on the risks of research participation is consistent with the goal of moving the IRB process of risk/benefit assessment from being entirely subjective to being evidence-based. Finally, recommendations are provided on how to approach these issues in IRB applications and investigators are called to help to build a corpus of scholarship that can advance empirical knowledge in this area.
\end{abstract}

Keywords: gay youth, homosexuals, lesbians, bisexuals, ethics, institutional review boards 


\section{INTRODUCTION}

Regulatory boards charged with protecting human research subjects, such as Institutional Review Boards (IRBs), have had an increasing influence on the conduct of research involving human subjects. Within the U.S., the IRB system is struggling to manage an expanding mission that extends beyond assessments of risk-benefit ratios to also sometimes include responsibility for managing conflicts of interest, compliance with regulations regarding accessing health records, assuring research protocols correspond to grant applications, and others (Gunsalus et al., 2007). At the same time, the scientific enterprise is becoming increasingly specialized, thereby diminishing the likelihood that any given IRB will have a member with expertise in the protocol under review. The implications of this so-called "mission creep" for researchers is that every aspect of the design and conduct of their study may be subject to modification by IRB members who are not subject-matter experts. Particularly in value-laden areas of scholarship, such as sexuality research, this creates the potential for individual values to threaten academic freedom and the quality and conduct of research that has the potential to improve sexual health.

The goals of this invited article are ambitious--a call to action to sexuality researchers to improve the IRB review of sexuality research by transforming it into an evidence-informed process. Transforming this process will require sexuality researchers to become experts in the ethical and regulatory issues involved in their research domain and to collect data on the risks and benefits to their participants. This article focuses on my own area of research on LGBT (lesbian-gay-bisexual-transgender) youth, but my aspiration is that the information and recommendations are more broadly useful to sexuality researchers. I begin by describing the need for sexuality research with LGBT youth, focusing specifically on HIV research with young men who have sex with men (MSM) as an example. Next, I illustrate the ethical and regulatory 
issues related to research with LGBT adolescents by using the IRB review of one of my own studies as a case example. This includes a review of the relevant U.S. federal and local regulations germane to the study. The intention of this policy review is not to provide an overview of every aspect of ethical and regulatory issues for research involving adolescents; such comprehensive reviews already exist (Field, Behrman, \& Institute of Medicine (U.S.) Committee on Clinical Research Involving Children, 2004; Santelli et al., 2003). Nor is the purpose to describe general concerns about dysfunction in the IRB review process; such critiques have also already been published (Fost \& Levine, 2007; Gunsalus et al., 2007). Instead, empirical data will be brought to bear on this issue. Specifically, analyses are conducted on how requiring parental consent for LGBT youth under age 18 may alter study findings and the conclusions on which they are based. Data are also presented on participants' appraisals of the risks associated with research participation. The goal of providing such empirical data is to help inform scholars and IRB members about the effects of requiring parental permission and potential psychological risks for LGBT youth in participating in research. The provision of this kind of empirical data on the risks of research participation is consistent with the goal of moving the IRB process of risk/benefit assessment from being entirely subjective to being evidencebased (Gunsalus et al., 2007; Wendler, Belsky, Thompson, \& Emanuel, 2005). Finally, recommendations are provided for investigators on how to approach these issues in their individual protocols and also how they can help to build a corpus of scholarship that can advance knowledge in this area.

\section{The Need for Sexuality Research with LGBT Youth}


Over the last 20 years, a few highly cited studies have suggested that lesbian, gay, and bisexual identified adolescents ${ }^{1}$ exhibit an array of health disparities relative to their heterosexual peers. Studies have identified similar disparities when the focus is on youth who are transgender, same-sex attracted, or engage in same-sex behavior (hereafter abbreviated LGBT for all such youth, while acknowledging the heterogeneity within this label). For example, in the 1990s, the Centers for Disease Control and Prevention (CDC) conducted a survey of nearly 3,500 men who have sex with men (MSM) ages 15-22 years across seven U.S. cities and documented a very high prevalence of HIV in this population (7.2\% overall; $14.1 \%$ in Black youth) (Valleroy, MacKellar, \& Karon, 2000). Current evidence indicates that each year there are nearly twice as many young MSM infected than there are young women infected through all risk categories (CDC, 2009). Despite this fact, there has not been commensurate attention to the HIV prevention needs of these young MSM (Mustanski, Garofalo, Herrick, \& Donenberg, 2007). In fact, several recent reviews that identified more than 60 published articles on randomized controlled trials or quasi-experimental studies on HIV risk reduction interventions for adolescents $(\sim$ age $<18)$ failed to report even one effective intervention targeted at adolescent MSM, while effective interventions have been described for many other populations at lower risk (Johnson, Carey, Marsh, Levin, \& Scott-Sheldon, 2003; Malow, Rosenberg, Donenberg, \& Devieux, 2006; Mullen, Ramirez, Strouse, Hedges, \& Sogolow, 2002; Pedlow \& Carey, 2004; Robin et al., 2004; Semaan et al., 2002). With most prevention funders requiring existing evidence of program effectiveness, the absence of such research on adolescent MSM effectively eliminates major

\footnotetext{
${ }^{1}$ The definition of the term adolescent varies somewhat depending on the medical, public health, or psychosocial context in which it is used. It is generally broadly considered the period of life from puberty to maturity. Here we are considering the period of middle to late adolescence, usually considered the ages of 14-16 and 17-20, respectively (Behrman, Kliegman, \& Jenson, 2004).
} 
sources of programmatic funding for this high risk group and thereby limits the ability to address these health disparities.

A lack of research on LGBT persons under age 18, relative to other groups of adolescents, has negative consequences beyond the ability to adequately address health disparities. Research on LGBT adolescents is critical to our basic understanding of diversity in the development of core aspects of sexuality, like sexual attractions and orientation, gender identity and expression, and romantic relationships. While the consequences of limited research with LGBT youth for basic and applied issues are clear, the cause is less well established. No studies of researchers or funders have been conducted to clearly establish the cause of less research in this area, but my experience from extensive discussions with sexuality and HIV researchers leads to an informed opinion that fear of, or experience with, an inability to obtain IRB approval for such studies is a major culprit (for documentation of such an experience, see Miller, Forte, Wilson, \& Greene, 2006). I have repeatedly heard from researchers that they planned to start their sample at age 18 because of the expectation that they could not get approval for waivers of parental consent for younger participants from their IRB or could not afford the time and funding required for a protracted review. Dovetailing with this anecdotal experience is broader data from an American Psychological Association survey of 411 psychologists who were recipients of National Institutes of Health (NIH) grants in 2004. Alarmingly, $42 \%$ of these senior investigators reported that, because of concerns about IRB rejection of their application, they modified their research plan; $20 \%$ reported they did not even pursue a particular line of research because of fears of IRB rejection of the application (Fendrich, 2009). These findings suggest a broader concern about self-censorship by investigators, but also lend credence to the anecdotal 
experience that investigators avoid including LGBT participants under the age of 18 because of concerns regarding IRB rejection of the protocol.

LGBT people do not emerge fully formed at age 18 like the Roman goddess Venus from the sea and it is not scientifically sound to begin all studies of LGBT populations at age 18. Waiting until LGBT persons turn 18 to engage them in research and then retrospectively report on experiences at earlier ages is an unsatisfactory alternative for several reasons. First, recall of past sexual events is subject to a number of forms of recall bias, such as telescoping and forgetting (Croyle \& Loftus, 1993). Second, the rapid change in the social status of homosexuality (Loftus, 2001) means even accurate retrospective reports of a current 18-year-old may not match the current experiences of a younger person. Third, there are large developmental changes in the context of one's life around age 18 in the U.S., meaning that research on participants over age 18 may not generalize to individuals even just a few years younger. These biases and limitations suggest that accurately understanding risk and resiliency processes in LGBT adolescents requires the participation of participants under the age of 18.

\section{Case Example}

Project Q2 was a longitudinal study of 246 LGBT youth ages 16-20 years at the baseline assessment (Mustanski, Garofalo, \& Emerson, 2010). At the time of initial IRB review, there were two aims of the study: (1) to identify the prevalence and predictors of mental health problems (particularly suicidality), substance use, and sexual risk taking among LGBT youth, and (2) to increase understanding of the development of sexual orientation. At the time of inception, the study was funded by the American Foundation for Suicide Prevention (Waves 1, 3, and 4) and through institutional support (Wave 2). While other sources of funding have subsequently been obtained to add further waves of data collection and expand the aims, as of 
yet Project Q2 has not been supported by any federal funds. The initial design planned for the use of Respondent Driven Sampling (Heckathorn, 1997), which involved peer recruitment to reach hidden populations. Data collection involved four waves of assessment across a one year period, including structured interviewer-administered psychiatric interviews, saliva samples for DNA extraction and genotyping (from those age 18 and older), completion of laboratory analogue risk tasks (e.g., Balloon Analogue Risk Task, Iowa Gambling Task), a family history interview, and audio computer-assisted self interviews (ACASI) measuring substance use, sexual risk taking, mental health, social relationships, sexual orientation and milestones, and personality, among others.

The project was conducted in collaboration with a community-based organization (CBO) with a long history of research with the LGBT community and the protocol was reviewed by both the University and CBO IRBs. The CBO was the primary site of data collection and their IRB had the most experiencing reviewing protocols focused on LGBT populations, so the protocol was first submitted to the CBO's IRB. After the protocol was approved by the CBO's IRB, it was then submitted for review at the University IRB. After six months and four rounds of correspondence and full board review, the protocol was approved by the University IRB pending receipt of a Federal Certificate of Confidentiality. ${ }^{2}$ One month later, the Certificate was received and then one additional month was required to obtain approval for the revised protocol with the CBO's IRB. In total, the process from initial submission to approval of the protocol took 10 months of a research study that was funded for two years.

\footnotetext{
${ }^{2}$ Certificates of Confidentiality are issued by the National Institutes of Health (NIH) to protect identifiable research information from forced disclosure. They allow the investigator and others who have access to research records to refuse to disclose identifying information on research participants in any civil, criminal, administrative, legislative, or other proceeding, whether at the federal, state, or local level.
} 
The majority of the correspondence with the University IRB was to respond to requests for more information on various details of the study and to insure that all regulatory and bureaucratic documentation was in place (e.g., "provide additional background/literature," "clarify the role of the co-investigator," and reconfirm that data will not be accessed from participants' medical records). Three domains were of particular relevance to research with LGBT adolescents and will be discussed in detail: waiving parental consent for participants under the age of 18 , risks related to participation, and implication of conducting research with a "vulnerable" population. When helpful, quotations from the IRB applications and determinations letters for Project Q2 are included along with a review of the relevant ethical and regulatory issues.

\section{Parental Permission for Adolescent Research Participation}

Our initial application for Project Q2 requested a waiver of parental permission for 16and 17-year-olds to participate (see Appendix 1 for the text of such a request). The initial IRB review response letter stated, "Regarding the requested waiver of Parental Permission for 16-17 year olds: The justification for the requested waiver in Appendix B and in your application is eloquent and well-grounded in the literature when considered in the context of minimal risk research." Based on this positive feedback, we made very few revisions to this section in the revised application. However, the next round of review of the protocol said:

Please provide further detail regarding the justification of a waiver of parental permission for the administration of survey instruments to 16-17 year olds within the context of the proposed research. The survey instruments submitted are used for mental health evaluation in this population and contain highly sensitive (and possibly incriminating) information, and parental consent is normally required if they are administered in a therapeutic or clinical setting.

Please provide an "appropriate mechanism for protecting children who will participate as subjects," under 45 CFR 46.408(c), so that a waiver of parental permission may be considered for 16-17 year old subjects. Suggested mechanisms used in similar situations 
include community advisory boards, with professionals and lay members representative of the diverse subject pool, and youth advocates.

The IRB response alluded to several important concepts that are delineated in regulatory guidance, including waiving of parental permission, the types of mental health instruments being used with a putative norm of parental consent in therapeutic/clinical settings, and the provision of a mechanism of "protecting children." In the U.S., the primary source of federal regulations regarding children's participation in research come from 45 CFR 46 (subpart D): Additional Protections for Children Involved as Subjects in Research. These regulations specify that when children are involved in research, the assent of the child and the permission of the parent(s) is required in place of the consent of the subjects.

The first step in applying these guidelines should be making a determination that participants meet the definition of being "children." Properly making this determination requires a more elaborate deliberation than crudely applying a universal age cutoff. 45 CFR 46.402 provides this definition: "Children are persons who have not attained the legal age for consent to treatments or procedures involved in the research, under the applicable law of the jurisdiction in which the research will be conducted." Both the Society for Adolescent Medicine (SAM) Guidelines for research with adolescents (Santelli et al., 2003) and an Institute of Medicine (IOM) report (Field et al., 2004) on research with children interpret this statement to mean that the definition of "children" is set based on local laws regarding the types of treatments or procedures involved in the research. As a result, the age at which a participant is considered a child and therefore requires parental permission to participate varies across jurisdictions and even within jurisdiction depending on the research procedures being used. As both the SAM and IOM reports review, few U.S. states have laws specifically pertaining to the age at which persons 
can consent to participate in research. In most states, the general age of majority ${ }^{3}$ is 18 , yet almost every state recognizes a younger age for persons to consent to general health care and/or specific aspects of physical, mental, and sexual health care (reviewed in Appendix B of IOM report). Furthermore, most states specify situations in which certain groups are emancipated (granted adult rights), such as military enlistment, marriage, pregnancy, and living independently. Considering these federal and state regulations and laws, the SAM guidelines assert, "Under this definition, adolescents who have reached the age of majority or the age of consent for general health care or are emancipated are not children. Likewise, adolescent minors, who are allowed to consent to treatments or procedures involved in the research, should not be considered children." Under federal regulations, if someone is not defined as a child, then there is no requirement for parental permission to participate in research.

I now consider the application of these regulations and guidelines in the context of Project Q2, which took place in the state of Illinois. While the state of Illinois had no law setting the age of consent for participation in research, a number of other laws were relevant to the interpretation of the definition of "children." The university IRB guidelines for research with children referenced 755 ILCS 5/11-1: The Illinois Uniform Transfer to Minors Act, which set the age of majority at 18 years for the ability to form a contract, deal with assets, hold property, and other business transactions. Other state laws are also germane, including 325 ILCS 17: The Children's Privacy Protection and Parental Empowerment Act, which defines a "child" as anyone under the age of 16 , who therefore cannot consent to the sale or purchase of their personal information. Other Illinois state laws allow anyone 12 or older to give consent to

\footnotetext{
${ }^{3}$ The age of majority is the age designated by state laws at which individuals are no longer under the legal control and responsibility of their guardian. Passing the age of majority allows individuals to engage in a number of specific actions they were previously prohibited from undertaking, such as driving a car, voting, entering into a contract, consuming alcohol, etc.
} 
medical care and counseling related to the diagnosis or treatment of HIV, a sexually transmitted disease, family planning, alcohol use/abuse, and drug use/abuse (Santelli et al., 2003, p. 356). Also of relevance, 405 ILCS 5 allowed for receipt of mental health care at age 12. None of these laws mandate the consent of parents/guardians, and some specifically prohibit informing parents without the adolescent's consent. Based on federal regulations, these state laws, and the principals laid out by the IOM and SAM, in the context of studies of these topics in the state of Illinois a child may be defined as someone under the age of 12 and therefore parental permission is not a requirement for participants of this age or older. In the case of Project Q2, the university IRB opted to review the protocol as though 16 and 17 year olds were considered children under the regulations.

When a determination is made that participants should be defined as children, federal regulations allow an IRB to waive parental permission (45 CFR 46.408(c)). Waivers of parental consent require that "...the research protocol is designed for conditions or for a subject population for which parental or guardian permission is not a reasonable requirement to protect the subjects (for example, neglected or abused children)..." As shown in the Appendix 1, we have proposed that parental permission for LGBT adolescents to participate in health research may not be a reasonable requirement to protect them because many of their parents may be unaware of their children's sexual orientation. Requiring participants to disclose their sexual orientation to their parents as a condition for research participation could actually increase the risk since parental abuse, rejection, and neglect has been documented as a result of disclosure for some youth (D'Augelli, Grossman, \& Starks, 2008; D'Augelli, Hershberger, \& Pilkington, 1998; Savin-Williams, 1994). 
Regulations state that waiving parental permission requires the provision of an "appropriate mechanism for protecting the children who will participate as subjects in the research.” As shown in Appendix 1, we have proposed the use of multiple mechanisms in our prior research. First, we have made formal assessments of decisional capacity prior to obtaining assent for 16-17 year old participants. Consistent with research by Dunn and Jeste (2001), the first step has involved a determination of the adolescent's understanding of the study goals after they have been explained. In step two, participants are asked questions designed to assess their capacity to understand, appreciate, reason with, and express a choice about participation using a modified version of the Evaluation to Consent Form (Dunn \& Jeste, 2001; Moser et al., 2002; The University of California at San Diego Task Force on Decisional Capacity, 2003). If interviewers have any doubts about decisional capacity, they are instructed to seek the consultation of the study principal investigator before proceeding. Second, our research aims and protocols have been developed in partnership with CBOs that have a mission of serving the LGBT community. Many of these protocols are then reviewed by the CBO's own IRB. Their board is composed of both professional and lay members who represent and have extensive experience with the LGBT community. This allows our protocols to be reviewed by a board with much more extensive LGBT competency than is likely to ever exist on a University IRB. Such an approach is consistent with the principals of Community-Based Participatory Research, which has been advocated as a strategy for conducting sexual health research with adolescents (Flicker \& Guta, 2008). Third, we have had a youth advocate on hand at all performance sites who is offered as an independent advisor to the youth about their decision to participate in the study. We have selected youth advocates that have experience and competence in working with LGBT youth, but are not funded by the project or report to the investigators in order to maintain their 
independence. The research aims and protocol are explained to the youth advocate and it is made clear to them that their role is to help the youth determine what is in their own best interest--not the best interest of the research project. It is worth noting that very few youth have expressed an interesting in conferring with the youth advocate to consider their participation. Fourth, for many studies, we have applied for and obtained a Federal Certificate of Confidentiality to assist in protecting the privacy of our participants. A certificate permits researchers to resist disclosures they may otherwise be compelled by law to make. According to NIH, a certificate allows disclosures, such as to protect the subject or others in cases of suspected or known child abuse, but these disclosures are deemed voluntary instead of mandated. These certificates have been found to be a deterrent against being compelled to release or identify participants; however their value may be dependent on an institutions willingness to defend against disclosure (Beskow, Dame, \& Costello, 2008). It is recommended that institutions provide formal guidance to the IRBs, investigators, and participants about their willingness to act in the protection of participants' privacy.

With these additional protections in place, the university IRB determined that the Project Q2 protocol satisfied 45 CFR 46.406: "Research involving a minor increase over minimal risk and having no direct benefit to the individual subjects, but likely to yield generalizable knowledge about the subjects condition." The "condition" in this case is presumed to be the health issues under investigation that are found more often among LGBT youth. With the protections noted in place, the board granted a waiver of parental permission.

It is important to point out that the goal of waiving parental permission is not to circumvent the authority of parents and their ability to look out for the wellbeing of their children. Instead, it is to allow for scientists to conduct research that could improve the health of 
adolescents in cases where parental permission is not a reasonable requirement to protect the participating youth. Conducting research in these situations advances the ethical principal of justice--one of the three pillars of ethical review laid out in the Belmont Report. ${ }^{4}$ Questions of justice center around what individuals and groups receive the benefits of research and bear any burdens. Justice is served when vulnerable groups are not exploited nor are some groups excluded from reaping the potential benefits of research. According to the IOM report (Field et al., 2004, Chapter 2), this principal was central to the successful argument for all NIH grant applications to address the inclusion of children. Bureaucratic actions that prevent or severely obstruct researchers from conducting studies that have realistic potential to improve the health of LGBT adolescents are inconsistent with the ethical principal of justice. Investigators should consider highlighting how their protocols are consistent with the promotion of justice in their applications. IRBs are encouraged to consider the important principal of justice when evaluating the extent to which their regulations, guidelines, and review procedures facilitate or obstruct research with LGBT youth.

A second basic principal of the Belmont Report is respect for persons, which includes treating participants as autonomous individuals capable of making informed decisions and also providing additional protections to those with diminished autonomy. The extent to which adolescents should be respected to make their autonomous decision about research participation can be guided by empirical research. The IOM (Field et al., 2004, Chapter 5) and SAM (Santelli et al., 2003) reports review the relevant literature on adolescent development and capacity to

\footnotetext{
${ }^{4}$ According to the IOM 2004 report, the Belmont Report is the most widely cited statement of ethical research principals and serves as the foundation for IRB regulations. The report came from the National Commission for the Protection of Human Subjects of Biomedical and Behavioral Research, which was commissioned by the U.S. Congress in 1974 to identify the basic principles for ethical research with human subjects and to create guidelines for the application of those principles.
} 
consent to research participation. For example, one early study of $9,14,18$, and 21 year-olds found that 14-year olds were as skilled as adults on multiple forms of competency related to consenting to research participation (Weithorn, 1983). Based on their review of this literature, the SAM report concludes, "For mid- and late adolescents (aged 14 years old or older), understanding of research and the cognitive ability to make decisions about research participation are similar to these abilities in adults." Similarly, the IOM report suggests that children under age 9 or 10 have limited abilities to understand the purpose, risks, and potential benefits of research participation, but that by age 14 or 15 adolescents differ little from adults in these respects (Fields et al., 2004, Chapter 5). These conclusions challenge the broad inclusion of adolescents as a group with diminished autonomy.

IRB communications during the review of Project Q2 described the LGBT population as "vulnerable" and therefore in need of additional protections. Neither the Belmont Report nor current federal regulations offer a definition for the term "vulnerable," but it is typically defined as a research participants' inability to make decisions that are in their own best interests (e.g., cognitively disabled individuals, children) and/or a power differential that subjects participants to potential coercion and undue influence by their superiors (e.g., students, employees, prisoners). Children, prisoners, pregnant women, and handicapped or mentally disabled persons are provided as examples of vulnerable categories of subjects, but LGBT individuals are not identified in the regulations as having diminished autonomy. While it is clear that LGBT individuals are at elevated risk for some negative health outcomes, to what extent do LGBT people as a group have reduced capacity to make decisions about research participation? To what extent do LGBT people require additional safeguards, such as someone appointed to represent their best interest and to protect them, from coercion by researchers? I know of no evidence that 
demonstrates such decisional impairment and I believe many LGBT individuals would be insulted to have it implied otherwise. Instead, I suspect that there may be a confusion on the part of some IRB members about the difference between a population being vulnerable to negative health outcomes and vulnerable to coercion by researchers. This confusion is unfortunate because the first type of vulnerability invokes the ethical principal of justice and the need for research in a particular population, whereas the second type of vulnerability invokes need to provide additional safeguards.

The third ethical principal of the Belmont Report is beneficence, which means protecting participants from harm and making efforts to secure their well-being. In social and behavioral research, this principal is usually applied by identifying and minimizing the risks to participating in the study. So what are the risks of LGBT adolescents participating in behavioral research? Based on a review of the research literature, the SAM guidelines dismiss the notion that surveys may harm adolescents by promoting or inducing unhealthy behavior (e.g., initiation of sexual behavior after completing a sexual health survey). The SAM guidelines conclude that the primary risks to adolescent participation in behavioral research, particularly survey research, are disclosure of sensitive information to others and negative psychological reactions to participating. This suggests the primary focus of a human subject protection review of a study with LGBT youth should be on the methods and abilities of the investigative team to protect the privacy and confidentiality of their participants and to respond to any adverse psychological reactions resulting from participation.

Approaches to protecting participants' privacy and confidentiality are well established (e.g., use of participant numbers instead of names), but less well characterized are the potential of psychological harm from participating in research. As Fendrich, Lippert, and Johnson (2007) 
point out, it has become commonplace for researchers to include a warning clause in studies of "sensitive" topics that participating could cause embarrassment and possibly distress. In the absence of empirical data on the probability and consequences of experiencing distress from answering sensitive questions, IRBs may incorrectly estimate the risks. Estimation of the risk level in answering "sensitive" questions is important not only so as to correctly communicate risks to potential participants, but also because 45 CFR 46 allows for protocols that are determined to be "minimal risk" to undergo an expedited or exempt review process. The regulatory definition of "minimal risk is the probability and magnitude of physical or psychological harm that is normally encountered in the daily lives, or in the routine medical, dental, or psychological examination of healthy persons." A cursory review of any standard psychological intake form would show questions about depression, suicide, substance use, and other aspects of mental health that are sometimes considered "sensitive." Fendrich (2009) considered questions about sexuality, pointing out that the millions of people who donate blood in the U.S. are required to answer a number of questions about their sexual behavior (e.g., "Have you ever had sexual contact with a prostitute or anyone who takes money or drugs or other payment for sex?”). This speaks to how answering questions about sexuality may be commonplace, but not the potential reactions to answering such questions. Limited research has been done on this topic. Two studies found that, among undergraduates, answering questions about sexuality did not lead to a significant change in measures of distress, even among those with a history of childhood sexual abuse (Rojas \& Kinder, 2007; Savell, Kinder, \& Young, 2006). More research is clearly needed on the reactions of participants to sexuality research so as to move determinations of risk levels from being purely subjective to being evidence-based. Empirical Data from Project Q2 
Details of the Project Q2 recruitment process and sample characteristics can be found elsewhere (e.g., Mustanski, Garofalo, \& Emerson, 2010), but briefly 246 ethnically diverse, 1620 year old LGBT, "queer," "questioning," and/or same-sex attracted youth living in the Chicago area participated in this study. Youth were recruited over 18 months from 2007-2008 from multiple sources, including e-mail advertisements, cards and flyers distributed in LGBTidentified neighborhoods and events, and through incentivized peer recruitment. The mean age of the sample was 18.31 years $(\mathrm{SD}, 1.32), 31 \%$ were under age $18,86 \%$ were racial/ethnic minorities ( $57 \%$ Black, $11.4 \%$ Latino), $49 \%$ were born male ( $51 \%$ female), $8 \%$ identified as transgender, and $62 \%$ of the sample identified as gay/lesbian (29\% bisexual, $9 \%$ other). Fiftythree percent of participants we recruited by another participant, $15 \%$ responded to flyers and Internet postings, $9 \%$ from community events, $14 \%$ from community-based agencies, and the remainder did not recall or report how they heard about the study.

The following analyses were limited to the 75 participants under the age of 18 in order to consider the effects of a parental consent requirement. In terms of living situation, $81 \%$ were living with their parents or family, $5 \%$ had no permanent address, and the remainder were living alone, with roommates, romantic partners, or in a group home. As preliminary data for a future study, participants were asked how they would feel if we asked for permission to contact their mother or father to be involved in research. Thirteen percent of participants responded they were not in contact with their mother, $36 \%$ were not in contact with their father, and $11 \%$ were not in contact with either parent. Among those who were in contact with each parent, Table 1 reports attitudes towards contacting each parent to engage them in research. Few LGBT youth reported positive attitudes towards involving their parents in the research process. Among those with the specified parent involved in their life, $36 \%$ showed various degrees of positive attitudes towards 
contacting their mother and $29 \%$ held positive attitudes towards contacting their father. Among the 46 youth in contact with both parents, the correlation between attitudes toward contacting mother and father was $.39, p<.01$, indicating a limited degree of correspondence in attitudes towards contacting each parent. Youth were also asked if they think they would consent to us contacting their mother/father to involve her/him in research. Among those in contact with their mother, $40 \%$ said they thought they would let us contact her to be involved in research (35\% overall). Among those in contact with their father, $25 \%$ said they thought they would let us contact him to be involved in research (16\% overall). Nearly half of participants who were in contact with at least one parent (46\%) thought they would consent to us contacting at least one of their parents.

We next considered how requiring contact with either parent would have altered our findings in terms of the health, social, and developmental factors that were the focus of Project Q2. Among the youth in contact with at least one parent $(\mathrm{N}=67)$, we tested differences in levels of outcomes based on if they would consent to us contacting a parent for research (see Table 2). There were no significant differences in these factors depending on if youth were living at home with their family. This null effect is meaningful in that some researchers or IRBs may propose that residence with parents should be used as a criterion to determine if parental permission should be required. For example, it could be proposed that parental permission should be required for youth living with a parent. These findings suggest this may not be a good criterion because youth living with a parent were not significantly more likely to want to have a parent involved in research.

Mental disorders and suicide attempts were assessed using the Diagnostic Interview Schedule for Children (DISC; Shaffer, Fisher, \& Lucas, 2004) and we describe in detail our use 
of this established structured psychiatric interview elsewhere (Mustanski et al., 2010). Youth who reported being likely to consent to involving their parents in research had nearly three times the odds of a suicide attempt in the past year, suggesting that requiring parental permission for research could tend to lead to a sample that over-represents youth who have made a suicide attempt. Youth agreeing to contact their parents for research were 56\% less likely to have met diagnostic criteria for Conduct Disorder. Although this difference was non-significant in our sample of 67, the effect size is meaningful and power analysis indicates would be significant at $p$ $<.05$ in a larger sample $(\mathrm{N}=334)$. There were only small and non-significant differences in past year Major Depression and Post-Traumatic Stress Disorder.

Family support was measured using the Multidimensional Scale of Perceived Social Support-Family Subscale (Zimet, Dahlem, Zimet, \& Farley, 1988) collected using Audio Computer-Assisted Self-Interview (ACASI). To ease interpretation, we $z$-score standardized the measure and then reported means among youth separately by if they would involve their parents in research. Youth higher in family support were significantly more likely to report they would consent to contacting their parents about research, which was a small-to-moderate effect size (.44). This finding suggests that requiring parental permission for research would tend to exclude youth who did not perceive their relationship with their family to be as supportive. Sexual risk and substance use behaviors were obtained using the AIDS Risk Behavior Assessment (ARBA). Youth who reported binge drinking in the past six months were significantly more likely to say they would be willing to consent to contacting their parents about research, but there were no significant differences in unprotected sex or cigarette use.

To further explore the possible effects of requiring parental consent, we tested demographic differences (race, sex, education, SES). The sample of youth under age 18 was 
primarily Black, which restricted the ability to test for demographic differences; therefore, the full sample of 18-20 year old participants was used for these analyses. In a logistic regression model controlling for age, participants who were racial/ethnic minorities (Black OR $=.47, p=$ .08 ; Latino/a $\mathrm{OR}=.41, p=.12$; relative to White $)$ and bisexual $(\mathrm{OR}=.48, p<.05$; relative to gay/lesbian) tended to be less likely to consent to involving parents in research. This suggests requiring parental consent would create a race/ethnicity-based barrier to research participation and would tend to underrepresent bisexual-identified youth. There were no significant differences in family SES, education, or sex.

This pattern of differences in health, social, and demographic variables suggests that requiring parental permission for research on these topics with LGBT youth will tend to change research findings. According to our comparisons, parental permission would tend to underrepresent youth who have not made a suicide attempt, have less family support, binge drink less, were racial/ethnic minorities, and identify as bisexual. As such, parental consent requirement will tend to underrepresent many of the youth who are most in need of the benefits of research, such as the development and validation of targeted health programs.

As part of the review process, IRBs must make determinations about the risks of research participation, how they have been minimized, and how they compare to research benefits. In almost all cases of social/behavioral research, this involves a subjective determination based on opinions about the probability of a risk outcome occurring and its likely consequences. Fendrich et al. (2007) have discussed the fact that IRBs frequently require the inclusions of "warning clauses" about potential adverse psychological reactions to surveys of drug use and sexual behaviors. For example, the IRB approved assent and consent documents for Project Q2 stated under the heading: What are the possible risks and discomforts? 
We will be asking you personal questions about your sex life, mental and emotional health, any alcohol or drugs you may have used, and potentially criminal behavior. Although this information is confidential, some of these issues could make you feel uneasy or embarrassed. If there are questions you do not want to answer, you do not have to do so. You can stop taking part at any time. A staff member will be available if you want to talk about anything that comes up during the interview. We will also refer you to counseling services if you want.

According to the results in the Fendrich et al. (2007) study of questions about drug use, such clauses may inappropriately convey an exaggerated sense of a survey's risks. Investigator and IRB appraisal of such psychological risks will necessarily continue to be subjective until empirical data are collected to help inform these decisions. At the one-year follow-up assessment of the Project Q2 sample, we included response probes based on the work of Fendrich et al. We did not request and obtain IRB approval until 40 of these interviews had already been completed, so only a sub-sample of 181 received these items (25 did not participate in the one year followup).

Table 3 contains responses from all Project Q2 participants to the questions, "We are trying to understand what it is like to participate in one of our research studies. How did you feel answering the questions about..." followed by each of the three domains of the study that are often considered "sensitive topics" by IRBs. Across these three domains, approximately $90 \%$ of participants said they were "very comfortable" or "comfortable" and at most 3\% of participants described themselves as "very uncomfortable." Our finding of very few participants reporting being very uncomfortable regarding answering these questions is similar to what has been found in the small number of other studies that have assessed reactions to questions about sexuality, substance use, and mental health (Fendrich et al., 2007; Jacomb et al., 1999; LanghinrichsenRohling, Arata, O'Brien, Bowers, \& Klibert, 2006). These findings call into question the opinion that answering questions about sexual behavior, substance use, and mental health often cause 
discomfort and the legitimacy and value of including cautionary statements in consent/assent documents. Such cautionary statements may instead misinform potential research participants about the potential risk/benefit ratio of their participation, which is inconsistent with the principal of informed consent/assent.

Comparisons regarding comfort across the three domains suggest that these participants were most comfortable answering questions about substance use compared to questions about sexual behavior and mental health and suicide, $\chi^{2}(2)=8.01, p>.05$, although these differences were relatively minor and comfort was high across all three domains. Just as the quality and truthfulness of participants answers to sensitive questions is likely to vary based on the methods used and settings in which data are collected (Tourangeau \& Yan, 2007), degree of comfort/ discomfort may also vary, and further research is needed do understand what aspects of study design make participants most comfortable.

When IRBs review research, an important determination is if the research should be classified as minimal risk. This determination is important not only because it establishes if the research can receive expedited review, but also because it alters the approvals that are needed for research with children (Field et al., 2004). Although efforts have been made to quantify the risks of children's daily lives and routine physical/psychological examinations (e.g., 27\% of children sometimes feel scared or afraid in daily life; Wendler et al., 2005), determinations regarding the minimal risk standard are largely made by IRBs using subjective opinions. We sought to inform this determination by asking participants, "Did you feel like answering any of these questions made you more uncomfortable than a typical visit to your physician, doctor, psychologist, or counselor." Of all Project Q2 participants, 11\% responded "Yes, more uncomfortable answering these questions," 54\% responded "No, it was more comfortable answering these questions," and 
$35 \%$ said "About the same." The application of our findings that the majority of participants were more comfortable participating in our study than a typical visit to their doctor is complicated by the fact that the standard is not explicit in term of the probability and magnitude of harm being greater for the majority of participants, most participants, a substantial proportion, or any participants. It could be argued that research participants do not have the knowledge and training to make risk comparisons, which is why these determinations are delegated to IRBs. This may be true, but nevertheless information from participants can be used to inform the deliberations of IRBs when making determinations about discomfort and risks. In the absence of data, IRBs may tend to overestimate the risks to subjects in social and behavioral research protocols, which may lead to unnecessary and time-consuming procedures. Overestimation of risk can also lead to implementation of putative protections that may be inappropriate, mitigate the scientific validity of the research, or discourage behavioral research involving certain populations. Collecting empirical data on perceptions of risk from participants could help tune these estimations made by IRBs. Ultimately, if the opinions of the IRB differ from the perceptions of participants themselves, it would seem appropriate for the IRB to provide the evidence used to arrive at their divergent opinion to investigators and potential participants. In addition to making determinations about risk, IRBs must make determinations about benefits and 45 CFR 46 specifies that a basic element of informed consent includes "A description of any benefits to the subjects or to others which may reasonably be expected from the research." While 45 CFR 46 does not define "benefit," the NIH Intramural Protocol Review Standards states that "A research benefit is considered to be something of health-related, psychosocial, or other value to an individual research subject [direct benefit], or something that will contribute to the acquisition of generalizable knowledge [social benefit]." Unfortunately, we 
did not collect research data on Project Q2 participants' perceptions of the direct benefits of their participation. However, many of the participants informally told the PI and staff that they felt it was helpful having someone listen to them talk about their experiences, that they felt validated, and that they had few other opportunities to engage in such beneficial discussions. IRB guidance does state that while "participation in research may be a personally rewarding activity or a humanitarian contribution, these subjective benefits should not enter into the IRB's analysis of benefits and risks" (Office for Human Research Protections). There is a distinction between feelings of making a humanitarian contribution by advancing research and when LGBT youth articulate that they believe they directly benefited from having a unique opportunity to talk about their life experiences in a supportive and accepting environment. However, the validity and implications of this difference has not been well studied. It seems clear that more systematic research is needed to enumerate and document the direct and immediate benefits of research participation. Doing so would give investigators more solid ground on which to base the content of their description of direct and social research benefits.

\section{Recommendations for Investigators}

In my experience conducting or collaborating on seven studies of LGBT youth (five that were federally funded), I have found that to successfully navigate the IRB review process required not only that my investigative team be familiar with relevant ethical, legal, and regulatory principals, but also that we articulate them in detail in our applications. We have needed to help educate our IRBs about federal and local regulations and how they should be applied to our work. We also need to, whenever possible, highlight the ethical mandate we have to conduct this research. Attempting this kind of education can be difficult and frustrating at institutions where IRB policies limit opportunities for bidirectional exchange between 
investigators and board members--often limiting communication to formal letters reporting the results of board review. A lack of transparency in the review process can prevent what ultimately could be a productive collaboration between investigators and IRBs together seeking to attain the most protection and benefits to research participants while advancing scientific knowledge. As a member of my own institution's IRB, I have become familiar with the kind of information that is most useful to board members when making these determinations in the absence of a direct dialogue with investigators, who are the subject matter experts. What follows are some recommendations for investigators. It is my hope that these recommendations may benefit investigators conducting research with LGBT youth and other forms of sexuality research, and may also help IRBs in the review of this work.

1. If possible, attend the meeting where your work will be reviewed. The NIH Intramural Research Branch invites investigators to participate in the initial review of their protocol so that they can answer board member questions and collaborate in setting appropriate protections and risk reduction procedures. One recent study found that, when a university changed its policies to encourage investigator attendance, protocols for which the investigator attended were reviewed in fewer days and with fewer meetings (Taylor, Currie, \& Kass, 2008).

2. Serve on your IRB. Being a member of your IRB means that you bring your expertise to the meeting and gives you a unique opportunity to educate the other board members about sexual health research, LGBT research, or research with adolescents.

3. Help advance knowledge about the risks and benefits of participating in research by asking participants how they felt about their participation in your study. Publish the results so as to build a corpus of knowledge that will allow for evidence-based determinations about risks 
and benefits. As scientists, we are in a unique position to bring to bear our expertise in understanding how people respond in various situations and we should harness this expertise to help inform the IRB review process. Whenever possible, provide your IRB the empirical data about risks and benefits of sexuality research as they may be unfamiliar with this area.

4. Most IRBs undergo continuing education. Suggest articles or offer to provide education that may inform the board about the risks and benefits of the kinds of research you conduct.

5. Become familiar with your local laws and regulations so that you can provide them, when necessary, to your IRB along with your application. Excellent sources of information about local laws about age to consent include the Guttmacher Institute website and Appendix B of the IOM report (Field et al., 2004). Local child advocacy organizations often maintain websites that contain information about laws pertaining to adolescent consent to healthcare and social services.

6. If your research focuses on populations that experience health disparities, it may be helpful to clearly articulate that while your population is vulnerable to negative health outcomes, this does not definitively cause them to have limited autonomy or be at risk for coercion and undue influence, and therefore they are not definitively a "vulnerable" group per regulations. This may help the board recall that the spirit of the vulnerable population provision in the guidelines is to provide extra protections to individuals that may not be able to freely provide fully voluntary informed consent, which is different than groups that experience health disparities. Confusing these two types of vulnerability can have the unintended negative consequence of providing the wrong kinds of protections to the wrong populations, thereby potentially further perpetrating inequities. 
7. When questions are raised during the IRB review that are not clearly articulated or you feel are sufficiently addressed in your application, request a meeting with the IRB staff for clarification. The staff that are responsible for writing these letters should be able to obtain clarification upon request from the investigator.

Appendix 1 includes the section of an IRB application that requests and justifies a waiver of parental permission for LGBT adolescents. It is provided as a framework that investigators can adapt to their own context when proposing research with LGBT youth. It may also provide useful information to investigators considering conducting sexual health-focused research with other adolescent populations.

\section{A Call to Action}

Investigators with the knowledge and skills to address some of the dramatic health disparities experienced by LGBT youth may feel an ethical mandate to conduct research that has the potential to improve the health and wellbeing of this community. In addition to the obstacles to successfully conducting any research project, there are additional roadblocks to doing work with LGBT youth (e.g., developing approaches to contacting them while maintaining their confidentiality). It can be extremely frustrating to agonize over perfecting every detail of a protocol to only then have it repeatedly questioned by anonymous individuals who may not have the same subject matter expertise. The standard IRB approach of written correspondence with long delays can be exasperating and is not designed to facilitate a collaborative relationship between IRBs and investigators. Investigators may be disturbed by requirements to make protocol changes that they perceive as decreasing the quality of the science without apparent reduction in risks or increasing protections. Communication can sometimes be unclear and, if a board has limited experience with the LGBT community, questions may seem uninformed, 
insensitive, or even homophobic. Community partnerships may be strained by the need for investigators to comply with IRB mandates over the recommendations of community members with expertise in serving the target population. Spiraling disgruntlement is not the solution.

As scientists, our best tool to improve the IRB review of our work is to conduct research that can help characterize the true risks to participation, document the benefits, and validate the methods. We need to build a corpus of research that will serve as a foundation for a data-driven IRB review of our work. The inclusion of questions about the risks and benefits of participating in studies should become standard. We need to document participant comfort with research participation, innumerate any negative consequences, and characterize the benefits. These findings need to be published and synthesized so that they can be included in IRB applications. Scientifically informed policy statements, such as the SAM guidelines referenced here (Santelli et al., 2003), should be drafted by scientific organizations that have an interest in facilitating high quality, ethically sound, and regulatory compliant sexuality research. Guidelines developed and endorsed by respected organizations can serve as critical sources of information for investigators and IRBs when reviewing sexuality-related protocols. This is a call to action to scientists to improve the quality of IRB review of research by laying the foundation to transform review from opinions about risks and benefits to data-driven informed decisions. 


\section{ACKNOWLEDGMENTS}

This article is based on a presentation at the meeting of the International Academy of Sex Research, San Juan, Puerto Rico, August 2009. Project Q2 was IRB approved by all engaged institutions. Use of quotations from correspondence with the IRB was officially determined not to be human subjects research by the University of Illinois at Chicago (UIC) IRB. Project Q2 was made possible by grants from the American Foundation for Suicide Prevention and the William T. Grant Foundation. I would like to acknowledge the contributions of Dr. Robert Garofalo and Ms. Erin Emerson, who served as Co-Investigators on Project Q2 and to the research team at UIC and Howard Brown Health Center. Thank you to Dr. Sarah Johnson, Dr. Robert Garofalo, Dr. Ritch Savin-Williams, and Ms. Erin Emerson who provided comments on drafts of the article. This manuscript is solely the responsibility of the author and does not necessarily represent the official views of the funding agencies or colleagues who provided feedback on the article. 
Appendix 1: Relevant Sections from an IRB application related to Waivers of Parental Consent XVII. Request for Waiver of Consent, Alteration of Consent, or Waiver of Documentation

An IRB may (1) approve a consent process that does not include, or alters, some or all of the elements of informed consent, or (2) the IRB may waive the requirement to obtain written consent (called a waiver of documentation), or (3) the IRB may waive the requirement to obtain informed consent entirely. In order to make these determinations, the IRB must ensure that the Federal requirements for each waiver/alteration criterion are met and justified for the specific research protocol.

A. Are you requesting a waiver of informed consent or an alteration of consent under 45 CFR 46.116 (d) for all or part of the research? $\square$ No $\bigotimes$ Yes

If YES are you requesting a:

$\bigotimes$ Waiver for all of the research $\square$ Waiver for recruitment purposes $\square$ An alteration of consent

If a waiver or alteration is not being requested, then please proceed to question $\mathrm{D}$ below in the application.

If you are requesting a waiver or alteration of consent, proceed to question B below. If you are requesting an alteration, also complete question $\mathrm{C}$.

In order to apply for a waiver or alteration of consent, you must provide protocol specific justification for the four following criteria. A waiver may be requested for the entire study or for only one portion of the research (for example: a waiver of informed consent is requested to identify potential research subjects from medical records, but informed consent is still be required for the later enrollment of the subjects for research participation-called a waiver for recruitment purposes). NOTE: If you are requesting a waiver of consent and accessing PHI, $a$ waiver of authorization is probably also required.

B. 1. Please provide a written explanation as to why you believe the proposed research (or portion of the research) will present no more than minimal risk to the subjects who participate:

We are requesting a waiver of parental permission for participants who are ages 16-17. Minor participants will be completing interviews on topics such as their sexual behavior, drug use, mental health, exposure to violence, relationships, and personal attributes. There is minimal risk that participants will feel a little awkward about completing these questionnaires. Participants can stop the interview at anytime without negative consequences or skip any question.

There is also the possibility of loss of confidentiality. We have taken steps to minimize this possibility (1) All identifying information will be kept in password protected file on a password 
protected server. Only project staff will have access to it. The password will be regularly changed and will be immediately changed if there is any staff turnover.(2) Questionnaire data will be stored using ID only (with the temporary exception of retaining contact information during the scheduling process). Data entered into computer files will be maintained on a subdirectory of a password protected server. (3) All data will be collected by well trained staff members who have been trained in the protection of human subjects and who have signed a confidentiality agreement.

We will also retained youth advisors, to act as youth advocates to assist youth in making decisions about participation and to make sure youth are not coerced into participating. The youth advisor will be a staff person who is not a staff member of the current project (to make them unbiased), but who is informed about the aims and methods of the study, and who has experience working with youth.

2. Please explain whether or not a waiver or alteration of informed consent would adversely affect the rights and welfare of subjects:

The formal evaluation of decisional capacity by the PI and/or trained staff and the inclusion of independent youth advocates will help assure that participants' rights are not violated. There is concern that the requiring of parental permission will put some youth at risk regarding disclosure of their sexual orientation to their parents. This may place these youth at risk for parental harassment, abuse or expulsion from the parental home.

3. Please explain whether or not it would be possible to conduct this research without a waiver or alteration of informed consent:

We believe that it would not be possible to complete this research without a waiver of parental permission for youth 16-17 years old. The costs and time associated with contacting the parents/guardians would be prohibitive. More importantly, in accord with national policy recommendations from the Society for Adolescent Medicine we believe requiring parental permission for the proposed study would have a number of possible negative effects, including: (1) reducing the validity of the findings by effectively eliminating potential participants unwilling to share permission forms with their parents/guardians; (2) increasing risk to some youth whose parents have a negative response to the material in the permission forms that would (correctly) suggest their child has a minority or alternative sexual orientation; and (3) adding little in the way of actual subject protection, given the minimal risk of participation in this study.

4. Please explain your plans, when appropriate, for providing any pertinent information to the subjects at a later date (e.g., after their participation in the study):

The fourth criterion for an alteration is appropriate under conditions in which information is deliberatively withheld from the research subjects in order to accomplish the intent of the study (e.g., psychological experiment with deception). However, this criterion would not apply to the current study since no information would be withheld.

C. If you are requesting an alteration of consent, please describe in detail how you wish to alter the consent process and justify the need for this alteration. 
We are altering the consent process for 16 and 17 year olds by waiving parental permission. Parental or guardian permission is not a reasonable requirement for our research with LGBQQ youth. Parental permission could put some youth at risk regarding disclosure of their LGBQQ identification to their parents. This may then place these youth at risk for parental harassment, abuse or expulsion from the parental home. Some of the adolescent participants may be homeless or accessing services at CBOs that provide HIV prevention services to adolescents (e.g., HIV testing, condoms) without parental knowledge. In these cases, it would be unreasonable to obtain parental consent primarily because adolescents would be unlikely to provide the name of their parent/guardian for fear of being returned home or punished. Additionally, attempting to contact a parent/guardian could place some youth at risk. Parental permission could not only place these youth at increased risk, but it would also substantially limit the generalizability of our research.

We have put in place several appropriate mechanisms for protecting the minors who participate in our research. (1) As part of the consent process, the RA will make a formal assessment of the each youth's decisional capacity to consent prior to obtaining written formed consent. The formal assessment of a youth's decisional capacity for participation in research will use a 2-step process. Consistent with research by Dunn and Jeste (2001), the first step will involve a determination of the participant's understanding of the study goals as previously explained by the RA during a review of the procedures. Youth will be asked, "Can you tell me what this study is about?" In step 2, potential subjects will be asked questions designed to assess their capacity to understand, appreciate, reason with, and express a choice about participation in our specific protocol. We will use a modified version of the Evaluation to Consent Form widely used by a number of university researchers for the determination of decisional capacity to consent for participation in research (Dunn \& Jeste, 2001; Moser et al., 2002; UCSD Task Force on Decisional Capacity, 2003). Participants will be asked to: (1) name things they will be expected to do during the study, (2) explain what they would do if they no longer wished to participate in the study, (3) explain what they would do if they experienced distress during the study and (4) identify potential risks for participating in the study. Respondents able to, in the judgment of the RA, communicate and give acceptable answers to these questions will be considered eligible to consent. Whenever the RA feels there is a question about the need for a more formal assessment of the decisional capacity of a potential participant he/she will be instructed to contact the PI before proceeding with the consent procedures. (2) There will always be a youth advocate on hand who is not involved in the research who will serve as an independent youth advisor. The advisors will be trained on the importance of their role and be given a brief overview of the study. After the study is explained to a potential participant, they will be given an opportunity to speak to the independent youth advisor before they make their decision to assent to participation.

The waiver is not inconsistent with Federal, State, or local law. 45 CFR 46.402(a) defines children as, "persons who have not attained the legal age for consent to treatments or procedures involved in the research, under the applicable law of the jurisdiction in which the research will be conducted." Illinois does not have a low about the legal age for consenting to research 
participation. We note that our youngest participants are age 16. In the state of Illinois persons as young as 12 are allowed to consent to STI and HIV testing and treatment, substance use/abuse treatment, and mental health care without requirement to notify parents (Field et al., 2004;

Guttmacher Institute, 2010). 


\section{References}

Behrman, R. E., Kliegman, R., \& Jenson, H. B. (2004). Nelson textbook of pediatrics (17th ed.). Philadelphia, PA: Saunders.

Beskow, L. M., Dame, L., \& Costello, E. J. (2008). Research ethics. Certificates of confidentiality and compelled disclosure of data. Science, 322, 1054-1055.

CDC. (2009). Surveillance summaries: Sexual and reproductive health of persons aged 10-24 Years--United States, 2002-2007. Morbidity and Mortality Weekly Report, 58.

Croyle, R., \& Loftus, E. (1993). Recollection in the kingdom of AIDS. In D. G. Ostrow \& R. C. Kessler (Eds.), Methodological issues in AIDS behavioral research (pp. 163-180). New York: Plenum Press.

D'Augelli, A. R., Grossman, A. H., \& Starks, M. T. (2008). Families of lesbian, gay, and bisexual youth: What do parents and siblings know and how do they react? Journal of GLBT Family Studies, 4, 95-115.

D'Augelli, A. R., Hershberger, S. L., \& Pilkington, N. W. (1998). Lesbian, gay, and bisexual youth and their families: Disclosure of sexual orientation and its consequences. American Journal of Orthopsychiatry, 68, 361-371

Dunn, L. B., \& Jeste, D. V. (2001). Enhancing informed consent for research and treatment. Neuropsychopharmacology, 24, 595-607.

Fendrich, M. (2009, August). Empirical research on risks from studies of sexuality and other sensitive topics: What we don't know may be hurting us. Paper presented at the meeting of the International Academy of Sex Research, San Juan, Puerto Rico.

Fendrich, M., Lippert, A. M., \& Johnson, T. P. (2007). Respondent reactions to sensitive questions. Journal of Empirical Research on Human Research Ethics, 2, 31-37. 
Field, M. J., Behrman, R. E., \& Institute of Medicine (U.S.). Committee on Clinical Research Involving Children. (2004). Ethical conduct of clinical research involving children. Washington, DC: National Academies Press.

Flicker, S., \& Guta, A. (2008). Ethical approaches to adolescent participation in sexual health research. Journal of Adolescent Health, 42, 3-10.

Fost, N., \& Levine, R. J. (2007). The dysregulation of human subjects research. Journal of the American Medical Association, 298, 2196-2198.

Gunsalus, C. K., Bruner, E. M., Burbules, N. C., Dash, L., Finkin, M., Goldberg, J. P., et al. (2007). The Illinois White Paper: Improving the system for protecting human subjects: Counteracting IRB "mission creep". Qualitative Inquiry, 13, 617-649.

Guttmacher Institute. (2010). State policies in brief: An overview of minors consent law. New York: Guttmacher Institute.

Heckathorn, D. D. (1997). Respondent-driven sampling: A new approach to the study of hidden populations. Social Problems, 44, 174-200.

Jacomb, P. A., Jorm, A. F., Rodgers, B., Korten, A. E., Henderson, A. S., \& Christensen, H. (1999). Emotional response of participants to a mental health survey. Social Psychiatry and Psychiatric Epidemiology, 34, 80-84.

Johnson, B. T., Carey, M. P., Marsh, K. L., Levin, K. D., \& Scott-Sheldon, L. A. (2003). Interventions to reduce sexual risk for the human immunodeficiency virus in adolescents, 1985-2000: A research synthesis. Archives of Pediatric and Adolescent Medicine, 157, 381-388. 
Langhinrichsen-Rohling, J., Arata, C., O'Brien, N., Bowers, D., \& Klibert, J. (2006). Sensitive research with adolescents: Just how upsetting are self-report surveys anyway? Violence and Victims, 21, 425-444.

Loftus, J. (2001). America's liberalization in attitudes toward homosexuality, 1973 to 1998. American Sociological Review, 66, 762-782.

Malow, R. M., Rosenberg, R., Donenberg, G., \& Devieux, J. G. (2006). Interventions and patterns of risk in adolescent HIV/AIDS prevention. American Journal of Infectious Disease, 2, 80-89.

Miller, R. L., Forte, D., Wilson, B. D., \& Greene, G. J. (2006). Protecting sexual minority youth from research risks: conflicting perspectives. American Journal of Community Psychology, 37, 341-348.

Moser, D. J., Schultz, S. K., Arndt, S., Benjamin, M. L., Fleming, F. W., Brems, C. S., et al. (2002). Capacity to provide informed consent for participation in schizophrenia and HIV research. American Journal of Psychiatry, 159, 1201-1207.

Mullen, P. D., Ramirez, G., Strouse, D., Hedges, L. V., \& Sogolow, E. (2002). Meta-analysis of the effects of behavioral HIV prevention interventions on the sexual risk behavior of sexually experienced adolescents in controlled studies in the United States. Journal of Acquired Immune Deficiency Syndrome, 30, S94-S105.

Mustanski, B. S., Garofalo, R., \& Emerson, E. M. (2010). Mental health disorders, psychological distress, and suicidality in a diverse sample of lesbian, gay, bisexual, and transgender youth. American Journal of Public Health, 100, 2426-2432. 
Mustanski, B., Garofalo, R., Herrick, A., \& Donenberg, G. (2007). Psychosocial health problems increase risk for HIV among urban young men who have sex with men: Preliminary evidence of a syndemic in need of attention. Annals of Behavioral Medicine, 34, 37-45.

Office for Human Research Protections (OHRP). IRB Guidebook. Retrieved 2/15/2010, from http://hhs.gov/ohrp/irb/irb_guidebook.htm

Pedlow, C. T., \& Carey, M. P. (2004). Developmentally appropriate sexual risk reduction interventions for adolescents: Rationale, review of interventions, and recommendations for research and practice. Annals of Behavioral Medicine, 27, 172-184.

Robin, L., Dittus, P., Whitaker, D., Crosby, R., Ethier, K., Mezoff, J., et al. (2004). Behavioral interventions to reduce incidence of HIV, STD, and pregnancy among adolescents: A decade in review. Journal of Adolescent Health, 34, 3-26.

Rojas, A., \& Kinder, B. N. (2007). Effects of completing sexual questionnaires in males and females with histories of childhood sexual abuse: Implications for institutional review boards. Journal of Sex and Marital Therapy, 33, 193-201.

Santelli, J. S., Smith Rogers, A., Rosenfeld, W. D., DuRant, R. H., Dubler, N., Morreale, M., et al. (2003). Guidelines for adolescent health research. A position paper of the Society for Adolescent Medicine. Journal of Adolescent Health, 33, 396-409.

Savell, J. K., Kinder, B. N., \& Young, M. S. (2006). Effects of administering sexually explicit questionnaires on anger, anxiety, and depression in sexually abused and nonabused females: Implications for risk assessment. Journal of Sex and Marital Therapy, 32, 161172.

Savin-Williams, R. C. (1994). Verbal and physical abuse as stressors in the lives of lesbian, gay male, and bisexual youths: Associations with school problems, running away, substance 
abuse, prostitution, and suicide. Journal of Consulting and Clinical Psychology, 62, 261269.

Semaan, S., Kay, L., Strouse, D., Sogolow, E., Mullen, P. D., Neumann, M. S., et al. (2002). A profile of U.S.-based trials of behavioral and social interventions for HIV risk reduction. Journal of Acquired Immune Deficiency Syndrome, 30, S30-50.

Shaffer, D., Fisher, P., \& Lucas, C. (2004). The Diagnostic Interview Schedule for Children (DISC). In M. Hersen (Ed.), Comprehensive handbook of psychological assessment (Chapter 21). Hoboken, NJ: John Wiley \& Sons.

Taylor, H. A., Currie, P., \& Kass, N. E. (2008). A study to evaluate the effect of investigator attendance on the efficiency of IRB review. IRB: Ethics and Human Research, 30, 1-5.

The University of California at San Diego Task Force on Decisional Capacity. (2003). Procedures for determination of decisional capacity in persons participating in research protocols. San Diego: University of California.

Tourangeau, R., \& Yan, T. (2007). Sensitive questions in surveys. Psychological Bulletin, 133, $859-883$.

UCSD Task Force on Decisional Capacity. (2003). Procedures for determination of decisional capacity in persons participating in research protocols. From http://irb.ucsd.edu/decisional.shtml

Valleroy, L. A., MacKellar, D. A., \& Karon, J. M. (2000). HIV prevalence and associated risks in young men who have sex with men: Young Men's Survey Study Group. Journal of the American Medical Association, 284, 198-204.

Weithorn, L. A. (1983). Children's capacity to decide to decide about participation in research. IRB: A Review of Human Subjects Research, 5, 1-5. 
Wendler, D., Belsky, L., Thompson, K. M., \& Emanuel, E. J. (2005). Quantifying the federal minimal risk standard: Implications for pediatric research without a prospect of direct benefit. Journal of the American Medical Association, 294, 826-832.

Zimet, G. D., Dahlem, N. W., Zimet, S. G., \& Farley, G. K. (1988). The multidimensional scale of perceived social support. Journal of Personality Assessment, 52, 30-41. 
Table 1: Attitudes towards contacting parents to participate in research among LGBT participants under age $18(\mathrm{~N}=75)$

\begin{tabular}{lcc}
\hline & $\begin{array}{c}\text { Mother } \\
(\mathrm{N}=65)\end{array}$ & $\begin{array}{c}\text { Father } \\
(\mathrm{N}=48)\end{array}$ \\
\hline Very Negative & $29 \%$ & $45 \%$ \\
Mostly Negative & $9 \%$ & $8 \%$ \\
Somewhat Negative & $9 \%$ & $12 \%$ \\
Neutral & $17 \%$ & $5 \%$ \\
Somewhat Positive & $11 \%$ & $12 \%$ \\
Mostly Positive & $8 \%$ & $12 \%$ \\
Very Positive & $17 \%$ & \\
\end{tabular}

Note: Attitudes were only reported if they were in contact with that parent. 
Table 2: Differences in health, social, and developmental outcomes depending on if youth (age < 18) were likely to consent to contacting either parent about research $(\mathrm{N}=67)$.

\begin{tabular}{|c|c|c|c|c|}
\hline & \multicolumn{2}{|c|}{$\begin{array}{l}\text { Likely to consent to } \\
\text { contacting parents for } \\
\text { research }\end{array}$} & \multirow[t]{2}{*}{$\begin{array}{c}\text { Test of } \\
\text { difference }\end{array}$} & \multirow[t]{2}{*}{$p$} \\
\hline & No & Yes & & \\
\hline Living with parents & $88.9 \%$ & $90.3 \%$ & $\mathrm{OR}=1.17$ & ns \\
\hline Past year Major Depression & $8.3 \%$ & $6.5 \%$ & $\mathrm{OR}=0.76$ & ns \\
\hline Past year PTSD & $13.9 \%$ & $12.9 \%$ & $\mathrm{OR}=.92$ & ns \\
\hline Conduct Disorder & $25.0 \%$ & $12.9 \%$ & $\mathrm{OR}=.44$ & ns \\
\hline Lifetime suicide attempt & $19.4 \%$ & $41.9 \%$ & $\mathrm{OR}=2.99$ & .05 \\
\hline Family support (standardized mean) & $\mathrm{M}=-.17$ & $\mathrm{M}=.27$ & $\mathrm{~T}=1.84$ & .07 \\
\hline $\begin{array}{l}\text { Unprotected vaginal/anal sex (ever } \\
\text { in past } 6 \text { months) }\end{array}$ & $25.7 \%$ & $22.9 \%$ & $\mathrm{OR}=.84$ & ns \\
\hline Smoke cigarette in past 6 months & $37.1 \%$ & $35.5 \%$ & $\mathrm{OR}=.93$ & ns \\
\hline Binge drink alcohol in past 6 months & $17.1 \%$ & $41.9 \%$ & $\mathrm{OR}=3.49$ & .03 \\
\hline
\end{tabular}

Note: Sample only includes those youth who were in contact with one or more of their parents. OR $=$ Odds Ratio. $T$-values are from independent samples. PTSD = Post-traumatic stress disorder. 
Table 3: Participants reactions to answering study questions among participants of all ages at 1year follow-up interview $(\mathrm{N}=181)$.

\begin{tabular}{lccc}
\hline & Sexual Behavior & Drug and Alcohol Use & $\begin{array}{l}\text { Mental health and } \\
\text { Suicide }\end{array}$ \\
\hline Very Comfortable & $39.8 \%$ & $51.4 \%$ & $44.2 \%$ \\
Comfortable & $49.2 \%$ & $39.2 \%$ & $44.2 \%$ \\
Uncomfortable & $8.3 \%$ & $6.6 \%$ & $9.4 \%$ \\
Very uncomfortable & $2.8 \%$ & $2.8 \%$ & $2.2 \%$ \\
\end{tabular}

